\title{
Estimation of Multiple Illuminants Based on Specular Highlight Detection
}

\author{
Yoshie Imai ${ }^{1,2}$, Yu Kato ${ }^{1}$, Hideki Kadoi ${ }^{1}$, Takahiko Horiuchi ${ }^{1}$, \\ and Shoji Tominaga ${ }^{1}$ \\ ${ }^{1}$ Graduate School of Advanced Integration Science, Chiba University, Japan \\ 2 Toshiba Corporation \\ \{yoshie.imai,yu_kato,kadoi\}@graduate.chiba-u.jp, \\ \{horiuchi, shoji\}@faculty.chiba-u.jp
}

\begin{abstract}
This paper proposes a method for estimating the scene illuminant spectral power distributions of multiple light sources under a complex illumination environment. The spectral power distributions including natural and artificial illuminants are estimated based on the image data from a high-dimensional spectral imaging system. We note that specular highlights on inhomogeneous dielectric object surfaces includes much information about scene illumination according to the dichromatic reflection model. First, we describe several methods for detecting specular highlight areas. We assume a curved object surface illuminated by multiple light sources from different directions. Then we estimate the illuminant spectrum of each light source from the image data of that highlight area. Based on this principle, we present an algorithm to estimate multiple illuminants. The feasibility of the proposed method is shown in experiments.
\end{abstract}

Keywords: Multiple light sources, dichromatic reflection model, specular highlight area, illuminant estimation.

\section{Introduction}

Estimation of scene illumination from image data has important imaging applications, including illumination design, color constancy, image processing, image rendering, and image retrieval. The scene illuminant estimation problem has a long history. In the past, many algorithms were proposed for scene illuminant estimation; however most algorithms assumed uniform illumination from a single light source [1-10]. It should be noted that our illumination environment is not necessarily a single light source such as daylight or a light bulb, but often consists of multiple light sources from different directions.

Recently, artificial light sources such as various fluorescent lights and light emitting diode (LED) are now in use in daily life. A complex illumination environment is constructed by the multiple illuminants including these artificial light sources and natural daylight.

This paper proposes a method for estimating the scene illuminant spectral power distributions of multiple light sources under a complex illumination environment. A highdimensional spectral imaging system is first realized using a liquid-crystal tunable filter, 
a monochrome CCD scientific camera with a cooling system, and a personal computer. We assume that objects captured by the imaging system in a scene are composed of inhomogeneous dielectric material like plastic or paint. In this case, the dichromatic reflection model suggests that light reflected from the object is decomposed into two additive components: the specular and the diffuse reflection components. The spectral power distribution of the specular component is coincident with the illuminant spectrum [2]; therefore the illuminant estimation can be reduced to detect the specular reflection component.

Moreover we note that, when a curved object surface is illuminated by multiple light sources from different directions, the illuminant spectrum estimated from each highlight area corresponds to each of the light sources. So the multiple illuminants can be estimated from different specular highlight areas on the curved object.

The first step of illuminant estimation is to detect highlight areas from the observed object image. Though many algorithms have been developed to detect highlights [11-14], in this paper we describe several detection methods: (1) use of variable thresholding of luminance, (2) use of luminance and hue components, and (3) use of a polarization filter.

The second step is to develop an algorithm for estimating the illuminant spectrum from the extracted highlight area. As mentioned above, the two-dimensionality of the object surface, the high-dimensional image data of the highlight area are projected onto a two-dimensional space spanned by the first two principal-components. Then the pixel distribution (histogram) in this space is divided into two straight clusters, corresponding to the specular and diffuse reflections. The directional vector of the specular highlight cluster is coincident to the vector of the light source. Therefore, the illuminant spectrum can be estimated by extracting the principal-component vector of the highlight cluster.

The feasibility of the proposed method is shown in experiments using plastic objects in a real scene. First, the performance of the highlight detection methods is compared in detail. Second, the accuracy of illuminant estimation is investigated in a complex environment using multiple light sources of LED, fluorescence, and daylight.

\section{Imaging System}

Figure 1 shows the camera system for spectral imaging system. A high-dimensional spectral imaging system is realized using a liquid-crystal tunable filter, a monochrome CCD scientific camera (Retiga 1300 - with a cooling system using a Peltier device), and a personal computer. We represent illuminant spectra with 69-dimensional vectors, where the visible wavelength range [400-700nm] is sampled at equal intervals of $5 \mathrm{~nm}$ and also sampled at eight wavelengths (404, 436, 488, 544, 584, 588, 612, $656 \mathrm{~nm})$ that correspond to peaks of general fluorescent spectra.

The total sensitivity function $R_{k}(\lambda)$ of the spectral camera system at $k$-th channel is computed by multiplying the transmittance $t_{k}(\lambda)$ of liquid-crystal tunable filter, the sensitivity function $r(\lambda)$ monochrome CCD scientific camera, and exposure time $e_{k}$ as shown below 


$$
R_{k}(\lambda)=e_{k} \cdot t_{k}(\lambda) r(\lambda),(k=1,2, \ldots, 69) .
$$

Figure 2 shows the total sensitivity function. The original CCD camera was modified so that the shutter speed could easily be changed from the outside computer. We justify exposure time $e_{k}$ so that the imaging system has constant sensitivity at each channel.

The spectral radiance of the reflected light (color signal) $Y(\lambda)$ from an object surface is described as

$$
Y(\lambda)=E(\lambda) S(\lambda)
$$

where $E(\lambda)$ is the spectral power distribution of the incident light to the object, and $S(\lambda)$ is the spectral reflectance of the surface. The sensor output $\rho_{k}$ of $k$-th channel is written as

$$
\rho_{k}=\int_{400}^{700} Y(\lambda) R_{k}(\lambda) d \lambda .
$$

If the band of the total sensitivity $R_{k}(\lambda)$ is narrow, the color signal can be obtained approximately as

$$
Y\left(\lambda_{k}\right)=\rho_{k} / \int_{400}^{700} R_{k}(\lambda) d \lambda,
$$

where $\lambda_{k}$ is the wavelength for $k$-th channel. So we interpolate $Y\left(\lambda_{k}\right)(k=1,2, \ldots, 69)$ to obtain the color signal $Y(\lambda)$ at arbitrary wavelength in the visible range. Thus, the color signal observed from each pixel point of an object surface can be estimated from the outputs of the imaging system by knowing the total spectral sensitivity functions $R_{k}(\lambda)$.

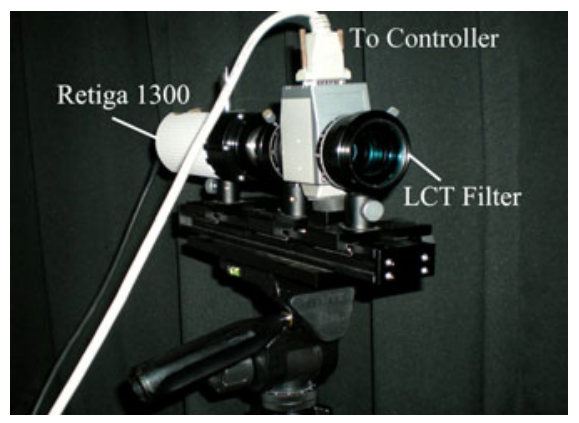

Fig. 1. Spectral imaging system 


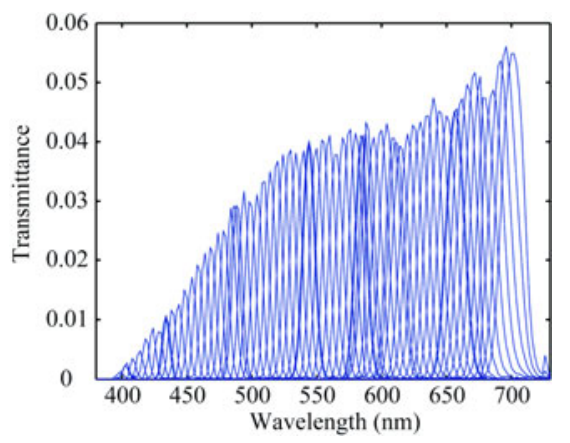

Fig. 2. Total sensitivity properties

\section{Detection of Highlight Area}

We consider three methods for detecting highlight areas on object surfaces.

\subsection{Detection Using Variable Luminance Thresholds}

The luminance value $Y$ for the color signal is computed as

$$
Y=\int_{400}^{700} Y(\lambda) \bar{y}(\lambda) d \lambda,
$$

where $\bar{y}(\lambda)$ is the luminance function which is one of the CIE color matching functions for the standard observers. A simple way for detecting highlight areas is to use a luminance threshold for the whole image area. It should be noted however, that the intensity of highlight on an object surface depends on the object color. Therefore the detection of multiple highlight areas is not always performed by simple thresholding if various object colors are contained in a scene.

In such a case, a method is proposed for repeatedly detecting multiple highlight areas by adaptively changing the luminance threshold. Figure 3 shows the procedure of this method. First, we calculate luminance value at every pixel and make the luminance histogram for the entire image. Second, the highlight candidates are extracted using a single threshold value, that corresponds to the luminance at a clear valley in the distribution shape of the luminance histogram. Third, the detected areas are labeled based on connectivity of pixels, so if the connected pixel count is small, the area is neglected. Then the connected regions with enough pixel counts are reserved as the target regions. Fourth, each target area is narrowed by increasing the threshold value, in order to determine precisely the highlight area. The above process is repeated for the respective target regions.

For instance, in Figure 3 (also see the color image in Figure 8), highlight areas on the blue cup is first detected with a low luminance threshold. Highlight areas on green and yellow objects are detected with higher luminance thresholds after four iterations of the above process. We can implement a semi-automatic thresholding technique. Thus, multiple highlight areas on different color objects are detected. 


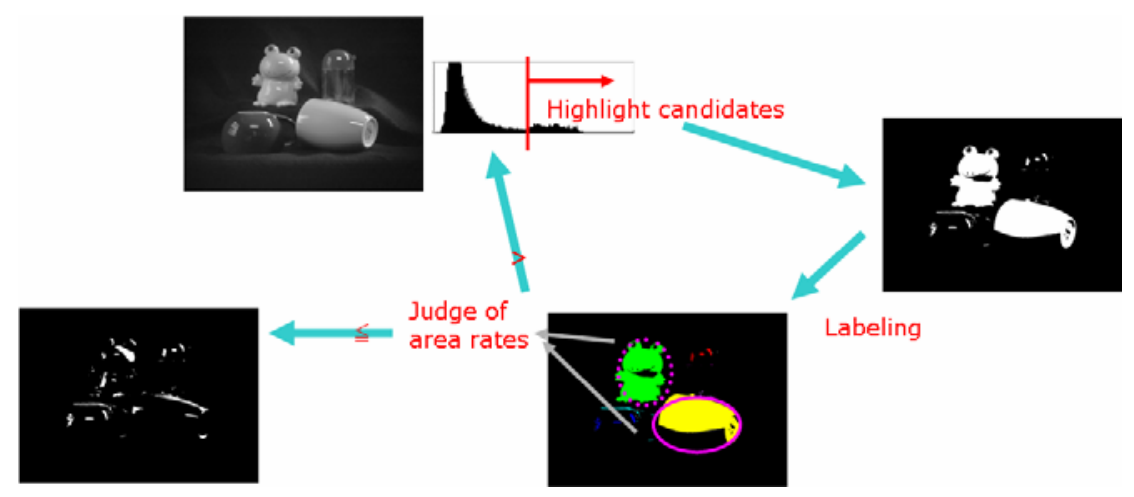

Fig. 3. Procedure of highlight area detection using variable luminance thresholds

\subsection{Detection Using Luminance and Hue Components}

The second method proposed for detecting highlight areas uses the luminance and hue components (Figure 4 illustrates an example of this method). We calculate the tristimulus values XYZ of the color signal by the color matching functions, and the chromaticity coordinates $(\mathrm{x}, \mathrm{y})$ as

$$
x=\frac{X}{X+Y+Z}, \quad y=\frac{Y}{X+Y+Z} .
$$

In this study, we define the hue $H$ as

$$
H=\arctan \left(\left(y-y_{0}\right) /\left(x-x_{0}\right)\right),
$$

where $\left(x_{0}, y_{0}\right)$ are standard white coordinates.

Normally the chromaticity coordinates of most scene illuminants are located closely to the chromaticity locus of the black-body radiator. Therefore the standard white coordinates $\left(x_{0}, y_{0}\right)$ should be placed at the black-body radiator locus on the xy diagram. Since the color temperature however, of scene illuminant is unknown, we select the suitable coordinates of $\left(x_{0}, y_{0}\right)$ in three ways, such as (1) $\left(x_{0}, y_{0}\right)=(1 / 3,1 / 3)$, (2) the average $(x, y)$ of all pixels by the Gray-World assumption [15], and (3) $(x, y)$ of brightest pixel by the White-Patch assumption.

We think that the hue of specular reflection part is different from surroundings as long as the light-source color is not the same hue as the object color when the specular reflection happens to the object usually. Therefore, high luminance area surrounded by different hue must be detected as a highlight area. The second method detects such area by setting a luminance threshold and hue thresholds. First, the candidates of highlight areas are detected by luminance thresholding. Second, the hue threshold of object color is determined by boundary color of the surface detected by the first luminance thresholding. By considering only high luminance area, the influence of the noise in the low luminance area can be eliminated. 


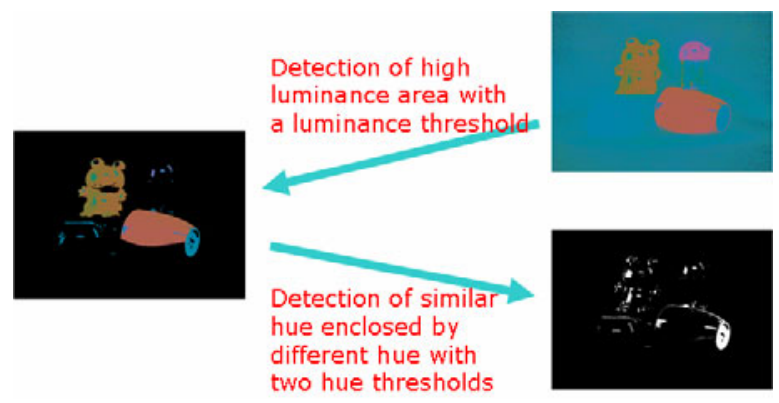

Fig. 4. Highlight area detection using luminance and hue components

\subsection{Detection Using a Polarization Filter}

The polarization property of light reflected from an object surface is available for highlight detection. When a polarization filter is attached to the front of the camera system, the linear polarization of the arbitrary direction can be observed by rotating the polarization filter. The proportion of the linear polarization element in the reflected light is represented by a polarization degree $\rho$

$$
\rho=\left(I_{\text {max }}-I_{\text {min }}\right) /\left\{I_{\text {max }}+\left(\frac{T_{p}(\lambda)}{T_{N}(\lambda)}-1\right) I_{\text {min }}\right\},
$$

where $T_{p}(\lambda)$ and $T_{N}(\lambda)$ are the spectral transmittances for linearly polarized light and unpolarized light respectively and $I_{\max }$ and $I_{\min }$ are the maximum and minimum intensities of reflected light, respectively [16]. Light reflected from an inhomogeneous dielectric is decomposed into two linear components: the diffuse reflection component and the specular reflection component. The diffuse component is not polarized and so invariant with polarization orientations, while the specular component is significantly polarized and varies greatly with polarization orientations. The polarization degree varies between 0 and 1 . One extreme $\rho=1$ represents the unpolarized state, such as reflection consisting of only the diffuse component and the other extreme $\rho=0$ represents the completely polarized state, such as pure specular reflection without the diffuse component.

Figure 5 depicts the transmitted radiance of light reflected from dielectric as a function of polarizer orientation. The transmitted light intensity oscillates sinusoidally between a minimum $I_{\min }$ and a maximum $I_{\max }$. For dielectrics this magnitude of oscillation is quite large in the specular highlight area, and then becomes smaller in the off-specular highlight area. Therefore, because the polarization degree $\rho$ is large for specular highlight area, we can detect the highlight area by thresholding of $\rho$ computed from all pixels. 


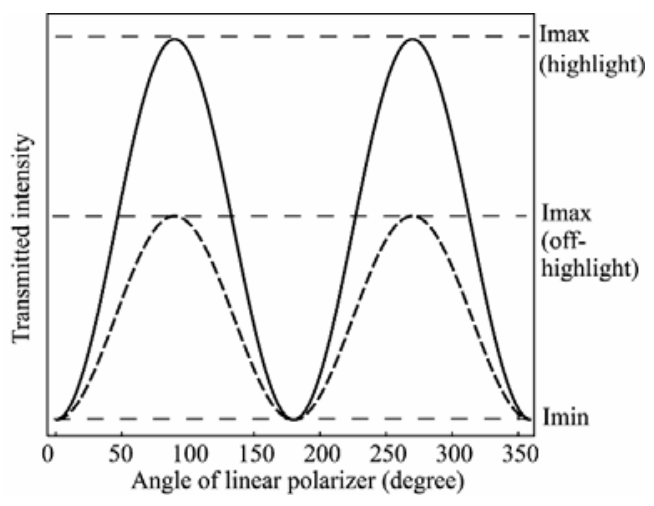

Fig. 5. Transmitted radiance of reflected light as a function of polarizer orientation

\section{Illuminant Estimation Algorithm}

The observed color signal from an inhomogeneous dielectric object is described by the dichromatic reflection model as follows:

$$
Y(\lambda)=Y_{S}(\lambda)+Y_{D}(\lambda)
$$

where the suffix $S$ and $D$ indicate the specular reflection component and the diffuse reflection component, respectively. To express the model in terms of the surfacereflectance function, let $S_{S}(\lambda)$ be the surface-spectral reflectance for the diffuse component, and let $E(\lambda)$ be the spectral power distribution of the incident light. Then the color signal is described as

$$
Y(\lambda)=S_{S}(\lambda) E(\lambda)+C E(\lambda)
$$

where $C$ is constant over the visible wavelength. Therefore the specular reflection component can be used for illuminant estimation.

Let $\mathbf{Y}$ be an n-dimensional vector of the camera output. As mentioned before, the surface with the specular reflection is two-dimensional. So for each highlight area the image data are projected onto a two-dimensional space spanned by two principal components. These components $\mathbf{p}_{1}$ and $\mathbf{p}_{2}$ are computed from the set of the $\mathbf{n}$ dimensional image data. Then we can derive the mapping equation:

$$
\left[\begin{array}{l}
C_{1} \\
C_{2}
\end{array}\right]=\left[\begin{array}{l}
\mathbf{p}_{1}{ }^{t} \\
\mathbf{p}_{2}{ }^{t}
\end{array}\right] \mathbf{Y},
$$

Figure 6 shows an example of pixel distribution (histogram) on the two-dimensional space $\left(C_{1}, C_{2}\right)$, projected from the image data of a highlight area. The pixel distribution in this space is divided into two straight clusters. One cluster corresponds to highlight pixels by specular reflection, and the other cluster corresponds to matte pixels by diffuse reflection. Therefore we note that the directional vector of the highlight cluster represents the illuminant vector. 
Estimation of the illuminant vector is reduced to finding the gradient of straight lines in the pixel cluster. Such a line component can be extracted by applying the principal component analysis or the Hough transform to the pixel distribution. We found that the line detection by the principal-component analysis is better in computational cost than the Hough transform.

Then, the illuminant vector $\mathbf{E}$ can be estimated by extracting the directional vector $\left(C_{1}{ }^{\prime}, C_{2}{ }^{\prime}\right)$ of the highlight cluster and transforming it inversely into the highdimensional spectral space as follows:

$$
\mathbf{E}=\left[\begin{array}{l}
\mathbf{p}_{1}{ }^{t} \\
\mathbf{p}_{2}{ }^{t}
\end{array}\right]^{+}\left[\begin{array}{l}
C_{1}{ }^{\prime} \\
C_{2}{ }^{\prime}
\end{array}\right],
$$

where + indicates a generalized inverse.

We note that, when an object with convex surface is illuminated by several light sources from different directions, the illuminant spectrum estimated from each highlight area corresponds to each of the light sources. That is, we assume that each highlight area is illuminated by only one source and not a combination of spectra power distributions of several light sources. Moreover, reflections generated by highlight areas do not interfere with other objects, i.e., that are not secondary sources.

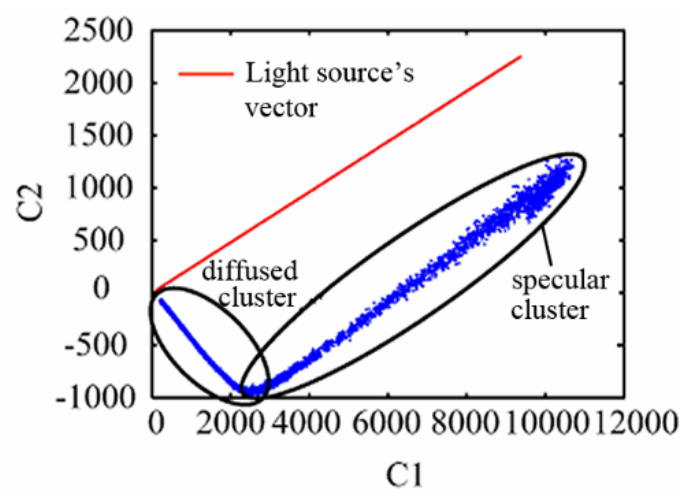

Fig. 6. Pixel distribution of image data on the two-dimensional space $\left(C_{1}, C_{2}\right)$

\section{Experimental Results}

We have examined the proposed method for estimating illuminant spectral power distribution under multiple light sources, based on the image data of the multiband imaging system. The feasibility of the method was examined in two steps: the highlight detection and the illuminant estimation.

\subsection{Experimental Conditions}

Figure 7 shows our experimental setup. Plastic objects placed on a table are illuminated with three different lightings: (1) daylight outside through the window, (2) fluorescent 
light source with D65 color temperature and (3) light emitting diode (LED) source. The multiband camera system aims from the front at the target object placed on the table. We also measure the illuminant spectra by using a reference white plate and a spectroradiometer (Photo Research, PR655). These measurements are used as the ground truth.

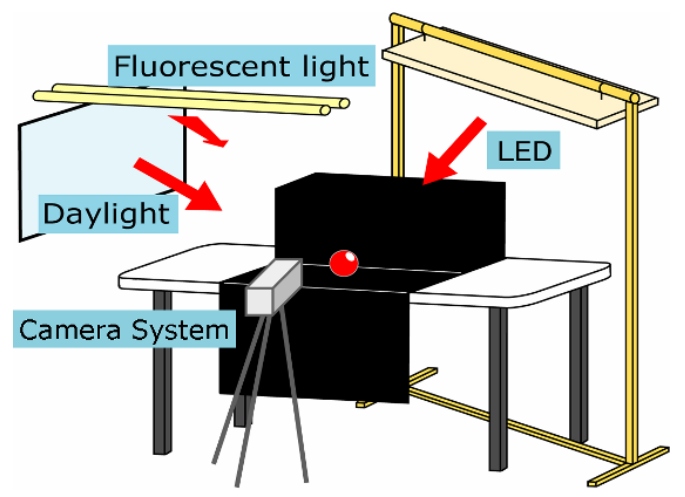

Fig. 7. Experimental setup

\subsection{Detection of Highlight Areas}

The scene in Figure 8 with different colored objects was used in experiments. Figure 9 shows bright areas detected by threshold of a low luminance value. We note that highlight areas are not properly detected by this simple thresholding.

Figure 10 shows the experimental results by the method (1) using the variable thresholding of luminance. The threshold of area counts (area rate) in Figure 10 (a) was set to $5 \%$ of all pixels. A highlight in the upper part of the yellow object cannot be appropriately detected. However, when we decrease the threshold in the recursive process, the detection accuracy improves as shown in Figure 10 (b) with the result by the threshold $0.3 \%$.

Figure 11 shows the results of the method (2) using luminance and hue components, where (a) is the result by the standard white coordinates $(1 / 3,1 / 3)$, (b) is the result by the Gray-World Assumption, (c) is the result by the White-Patch Assumption. The detection of highlight areas is influenced by object color. Three binary images in Figure 11 (a)-(c) are combined into Figure 11 (d). Look at the white bottom of the yellow cup. The entire white area is detected wrong as a highlight area.

Figure 12 shows the results by the method (3) using a polarization filter. Highlight areas on yellow and green objects are not properly detected. The image of polarization degree is depicted in Figure 12 (a), where image intensity indicates the value of polarization degree. Therefore, the highlight or glossy areas with specular reflection correspond to brighter areas in the image. It should be noted that the polarization degree depends greatly on the angle of incidence to object surface. As the angle is distant from the Brewster angle, the polarization degree decreases. No highlight area is detected on the yellow cup and green frog. 
Table 1 summarizes the detection characteristics of the three methods (1)-(3). Most highlight areas are extracted by (1). However, highlight on white object surface can not be detected sometimes by the method. In addition, it is necessary to examine application to the object with the pattern. The advantage of (3) using polarization is independence of object color. Therefore this method is applicable to colored texture

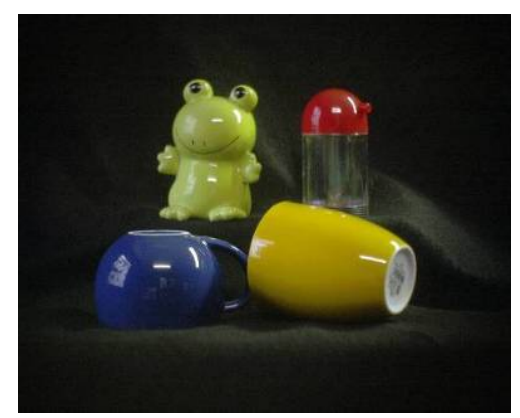

Fig. 8. Scene with different colored objects used in experiments

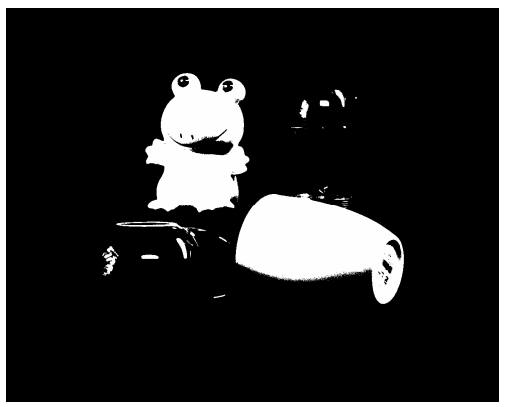

Fig. 9. Areas detected by threshold of a low luminance value

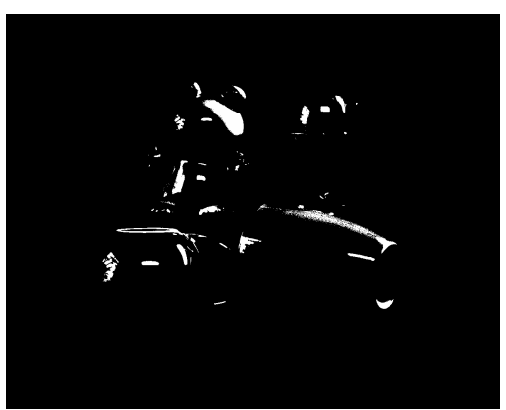

(a) Area rate threshold $5 \%$

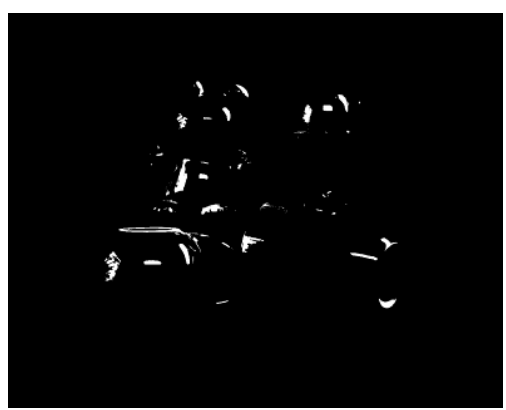

(b) Area rate threshold $0.3 \%$

Fig. 10. Detected highlight areas by using the variable thresholding of luminance 
surface. However the polarization degree depends on the angle of light incidence. That is, the method is unstable, depending on illumination geometry. Moreover rotation of the filter takes time, and the images captured with different rotation angles require much memory storage.

Thus it is suggested that the method (1) is the most suitable for detecting highlight areas by a normal imaging system.

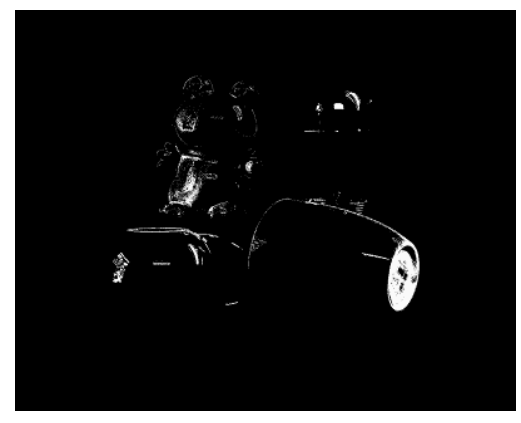

(a) White coordinates $(1 / 3,1 / 3)$

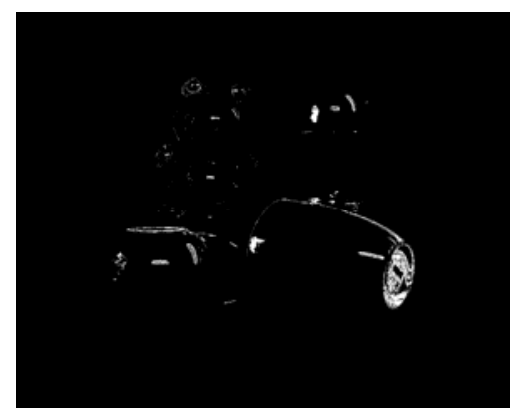

(c) White-Patch Assumption

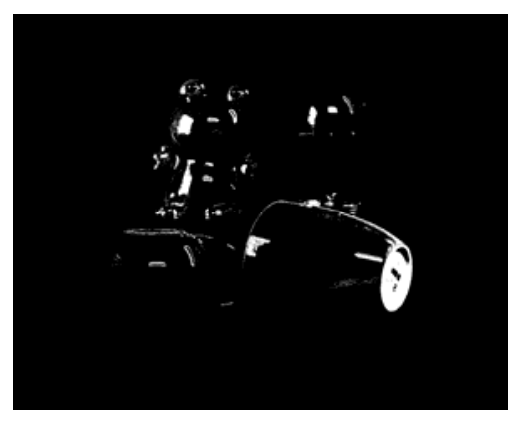

(b) Gray-World Assumption

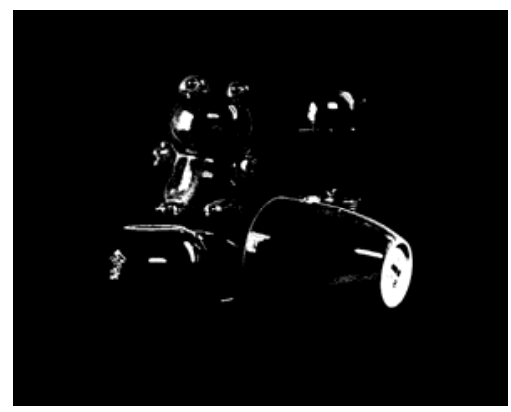

(d) Combined image of (a)-(c)

Fig. 11. Detected highlight areas by using luminance and hue components

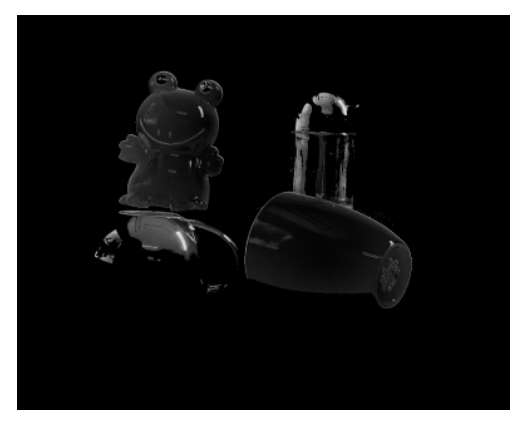

(a) Image of polarization degree

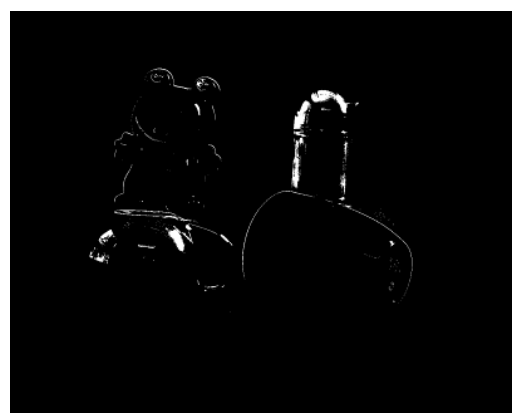

(b) Thresholding of polarization degree

Fig. 12. Detected highlight areas by using a polarization filter 
Table 1. Comparison of detection characteristics among three methods

\begin{tabular}{|c|c|c|c|c|c|c|}
\hline $\begin{array}{l}\text { Detection } \\
\text { method }\end{array}$ & $\begin{array}{l}\text { White } \\
\text { object } \\
\text { surface }\end{array}$ & $\begin{array}{l}\text { Colored } \\
\text { texture } \\
\text { surface }\end{array}$ & $\begin{array}{l}\text { Measurement } \\
\text { time }\end{array}$ & $\begin{array}{l}\text { Memory } \\
\text { capacity }\end{array}$ & $\begin{array}{l}\text { Computation } \\
\text { time }\end{array}$ & $\begin{array}{l}\text { Illumination } \\
\text { geometry }\end{array}$ \\
\hline $\begin{array}{l}\text { (1) } \\
\text { variable } \\
\text { thresholding }\end{array}$ & $\checkmark$ & & $\checkmark \checkmark$ & $\checkmark \checkmark$ & $\checkmark$ & $\checkmark \checkmark$ \\
\hline $\begin{array}{l}\text { (2) } \\
\text { luminance\& } \\
\text { hue }\end{array}$ & & & $\checkmark \checkmark$ & $\checkmark \checkmark$ & $\checkmark \checkmark$ & $\checkmark \checkmark$ \\
\hline $\begin{array}{l}\text { (3) } \\
\text { polarization }\end{array}$ & $\checkmark \checkmark$ & $\checkmark \checkmark$ & & & & \\
\hline
\end{tabular}

\subsection{Estimation of Illuminant Spectra}

Figure 13 (a) shows a part of the scene in Figure 3. Figures 13 (b)-(d) are the illuminant estimation results by the proposed algorithm. The blue curve in each figure is the estimated spectral power distribution, and the red curve is the direct measurement by a spectro-radiometer.

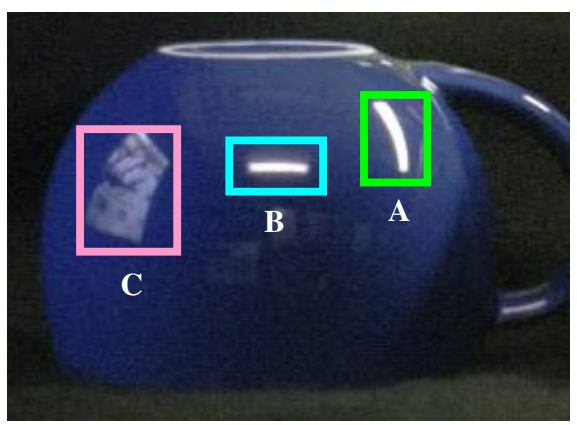

(a) Detected highlight areas

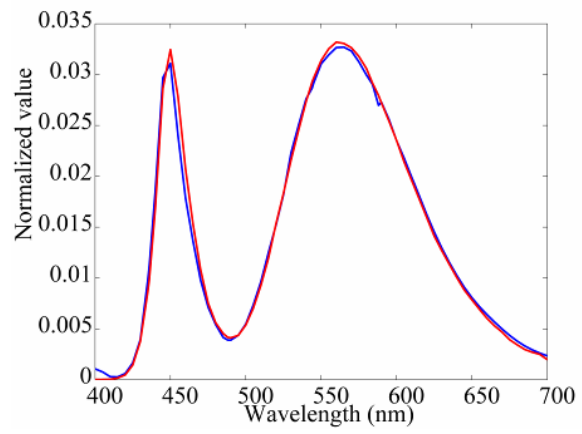

(c) Estimated result for area B

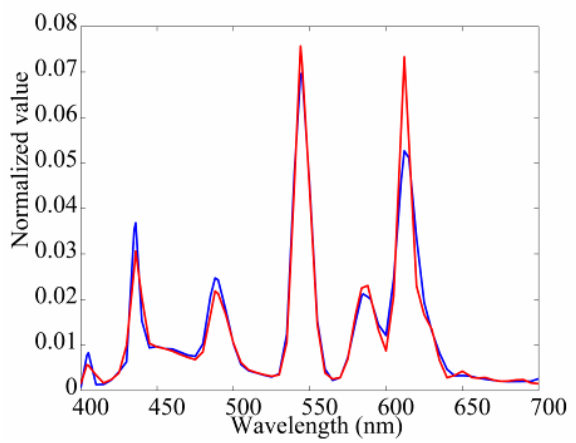

(b) Estimated result for area $\mathrm{A}$

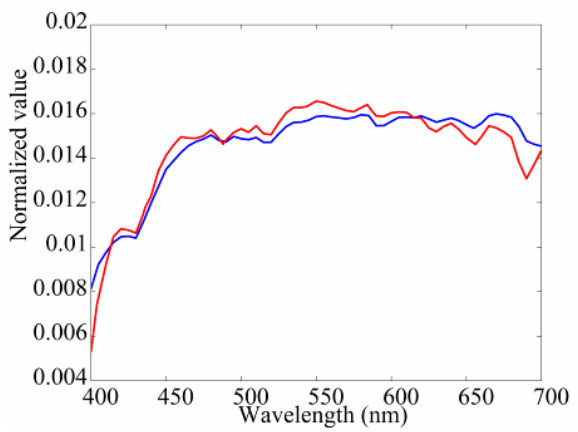

(d) Estimated result for area $\mathrm{C}$

Fig. 13. Experimental results of illuminant estimation of multiple light sources 
Three light sources illuminate the surface of a blue cup from different directions. In this case, three highlight areas correspond to the three light sources. The ability of estimating multiple illuminants is based on this fact.

The area A consists of fluorescent light with strong spikes. We can identify the fluorescent light source by knowing the wavelengths of the spike peaks. The area B provides us a LED light source. Moreover, notice that the area $\mathrm{C}$ reflects the outside scene through a window. We can estimate the daylight spectrum of natural light source from this highlight area.

We see that the estimated spectral curves of the respective light sources are very close to the direct measurements. These results suggest the feasibility of the proposed illuminant estimation method.

Moreover, we examined illuminant estimation from an object with color texture. In this case, when the highlight area on the object surface included the color texture, the estimation accuracy of illuminant spectrum decreased, compared with an object surface with uniform color.

\section{Conclusion}

We have proposed a method for estimating the scene illuminant spectral power distributions of multiple light sources under a complex illumination environment. The spectral power distributions were estimated based on the image data from a highdimensional spectral imaging system. Since specular highlights on inhomogeneous dielectric object surfaces included much information about scene illumination according to the dichromatic reflection model, we proposed three methods of highlight detection: (1) use of variable thresholding of luminance, (2) use of luminance and hue components, and (3) use of a polarization filter. Then we estimated the illuminant spectrum of each light source from the image data of that highlight area based on assumption that a curved object surface illuminated by multiple light sources from different directions. The feasibility of the proposed method was shown in experiments using plastic objects in a real scene. The experimental results suggested that the method of highlight detection using the variable thresholding of luminance was the most suitable for detecting highlight areas by a normal imaging system. These results also showed the accuracy of the estimated illuminant spectral power distributions of the respective light sources. Further examination of highlight detection of objects with texture remains as a future work.

\section{References}

1. Tominaga, S.: Multichannel vision system for estimating surface and illumination functions. J. Optical Society of America A 13(11), 2163-2173 (1996)

2. Tominaga, S., Wandell, B.A.: Standard surface-reflectance model and illuminant estimation. J. Optical Society of America A 6(4), 576-584 (1986)

3. Maloney, L.T.: Evaluation of linear models of surface spectral reflectance with small numbers of parameters. J. of the Optical Society of America A 3(10), 1673-1683 (1986)

4. Maloney, L.T., Wandell, B.A.: Color constancy: a method for recovering surface spectral reflectance. J. Optical Society of America A 3(1), 29-33 (1986) 
5. Tominaga, S., Haraguchi, H.: Estimation of fluorescent scene illuminant by a spectral camera system. In: Color Imaging X: Processing, Hardcopy, and Applications, San Jose, Calif, USA. Proceedings of SPIE, vol. 5667, pp. 128-135 (2005)

6. Tominaga, S.N., Tanaka, N.: Feature article: omnidirectional scene illuminant estimation using a mirrored ball. Journal of Imaging Science and Technology 50(3), 217-227 (2006)

7. Schultz, S., Doerschner, K., Maloney, L.T.: Color constancy and hue scaling. Journal of Vision 6(10), 1102-1116 (2006)

8. Tominaga, S.: Estimation of composite daylight-fluorescent light components based on multi-spectral scene images, In: Proceedings of the 14th IS\&T/SID Color Imaging Conference, Scottsdale, Ariz, USA, pp.125-130 (2006)

9. van de Weijer, J., Gevers, T., Gijsenij, A.: Edge-based color constancy. IEEE Transactions on Image Processing 16(9), 2207-2214 (2007)

10. Zhou, W., Kambhamettu, C.: A unified framework for scene illuminant estimation. Image and Vision Computing 26(3), 415-429 (2008)

11. Klinker, G.J., Shafer, S.A., Kanade, T.: The Measurement of Highlights in Color Images. International Journal of Computer Vision 2(1), 7-26 (1992)

12. Tan, R.T., Nishino, K., Ikeuchi, K.: Separating Reflection Components Based on Chromaticity and Noise Analysis. IEEE Transaction on Pattern Analysis and Machine Intelligence 26(10), 1373-1379 (2004)

13. Xu, S.C., Ye, X., Wu, Y., Zhang, S.: Highlight detection and removal based on chromaticity. In: Kamel, M.S., Campilho, A.C. (eds.) ICIAR 2005. LNCS, vol. 3656, pp. 199206. Springer, Heidelberg (2005)

14. Angelopoulou, E.: Specular Highlight Detection Based on the Fresnel Reflection Coefficient. In: IEEE 11th International Conference on Computer Vision, pp. 1-8 (2007)

15. Buchsbaum, G.: A spatial processor model for object colour perception. J. Franklin Institute 310(1), 1-26 (1980)

16. Tominaga, S., Kimachi, A.: Polarization imaging for material classification. Optical Engineering 47(12), 123201 (2008) 